\title{
A further generalization of certain integral inequalities similar to Hardy's inequality
}

Shanhe $\mathrm{Wu}^{\mathrm{a}, *}$, Banyat Sroysang ${ }^{\mathrm{b}}$, Shuguang $\mathrm{Li}^{\mathrm{a}}$

${ }^{a}$ Department of Mathematics, Longyan University, Longyan, Fujian, 364012, P. R. China.

${ }^{b}$ Department of Mathematics and Statistics, Thammasat University, Pathumthani, 12121, Thailand.

Communicated by R. Saadati

\begin{abstract}
In this paper, we investigate certain integral inequalities similar to Hardy's inequality. By introducing a monotonous function, we establish generalized versions of some known results related to the Hardy's inequality and give some new integral inequalities of Hardy-type. (C)2016 All rights reserved.
\end{abstract}

Keywords: Hardy's inequality, Hölder's inequality, similar version, generalization, integral inequalities. 2010 MSC: 26D15, 26D10.

\section{Introduction}

In 1920, Hardy presented the following famous inequality

$$
\int_{0}^{\infty}\left(\frac{F(x)}{x}\right)^{p} d x \leq\left(\frac{p}{p-1}\right)^{p} \int_{0}^{\infty} f^{p}(x) d x
$$

where $p>1, f$ is a nonnegative integrable function and

$$
F(x)=\int_{0}^{x} f(t) d t
$$

for all $x>0$. Furthermore, the constant $(p /(p-1))^{p}$ in 1.1$)$ is the best possible.

As is known, the inequality (1.1) is called Hardy's inequality (see [3], [5] and a historical survey paper [8]).

\footnotetext{
${ }^{*}$ Corresponding author

Email addresses: shanhewu@gmail.com (Shanhe Wu), b.sroysang@gmail.com (Banyat Sroysang), shuguanglily@sina.com (Shuguang Li) 
The Hardy's inequality has many applications in analysis and in the theory of differential equations (see, e.g., [12], [13] and [16]). This remarkable inequality has evoked the interest of many mathematicians and has received considerable attention from researchers. A number of papers which deals with the generalizations, improvements and variations of the Hardy's inequality have appeared in the literature, see [1, 2, 6, 7, 11, 17, 18, 27, 28, and the references therein.

The purpose of this paper is to discuss certain integral inequalities similar to Hardy's inequality. We begin by recalling some related results which were reported in the previous literature.

In 1928, Hardy [4] gave a generalized form of inequality (1.1) as follows

$$
\int_{0}^{\infty} \frac{F^{p}(x)}{x^{r}} d x \leq\left\{\begin{array}{l}
\left(\frac{p}{r-1}\right)^{p} \int_{0}^{\infty} x^{p-r} f^{p}(x) d x \text { for } \quad r>1 \\
\left(\frac{p}{1-r}\right)^{p} \int_{0}^{\infty} x^{p-r} f^{p}(x) d x \text { for } r<1
\end{array}\right.
$$

where $p>1, f$ is a nonnegative integrable function and

$$
F(x)= \begin{cases}\int_{0}^{x} f(t) d t \quad \text { if } \quad r>1 \\ \int_{x}^{\infty} f(t) d t \quad \text { if } \quad r<1\end{cases}
$$

for all $x>0$.

In particular, a straightforward consequence of inequality $(1.2)$ yields the following inequality

$$
\int_{0}^{\infty}\left(\frac{F(x)}{x}\right)^{p} x^{\alpha} d x \leq\left(\frac{p}{p-1-\alpha}\right)^{p} \int_{0}^{\infty} f^{p}(x) x^{\alpha} d x
$$

where $p>1+a, f$ is a nonnegative integrable function and

$$
F(x)=\int_{0}^{x} f(t) d t
$$

for all $x>0$.

For some results similar to the Hardy's inequality, we refer to [10, 14, 15, 19, 20, 23, 24].

In 1964, Levinson [9] established the inequality concerning integration from $a$ to $b$ as follows.

$$
\int_{a}^{b}\left(\frac{F(x)}{x}\right)^{p} d x \leq\left(\frac{p}{p-1}\right)^{p} \int_{a}^{b} f^{p}(x) d x
$$

where $0<a<b<\infty, f \geq 0, p>1$, and

$$
F(x)=\int_{a}^{x} f(t) d t
$$

for all $x>a$. 
In 2012, Sulaiman [21] proved two interesting inequalities similar to Hardy's inequality, as follows

$$
p_{1} \int_{a}^{b}\left(\frac{F(x)}{x}\right)^{p_{1}} d x \leq(b-a)^{p_{1}} \int_{a}^{b}\left(\frac{f(x)}{x}\right)^{p_{1}} d x-\int_{a}^{b}\left(1-\frac{a}{x}\right)^{p_{1}} f^{p_{1}}(x) d x
$$

and

$$
p_{2} \int_{a}^{b}\left(\frac{F(x)}{x}\right)^{p_{2}} d x \geq\left(1-\frac{a}{b}\right)^{p_{2}} \int_{a}^{b} f^{p_{2}}(x) d x-\frac{1}{b^{p_{2}}} \int_{a}^{b}(x-a)^{p_{2}} f^{p_{2}}(x) d x
$$

where $f>0$ on $[a, b] \subseteq(0, \infty), 0<p_{2}<1 \leq p_{1}$, and

$$
F(x)=\int_{a}^{x} f(t) d t, \quad x \in[a, b] .
$$

In 2013, Sroysang [22] generalized the inequalities (1.5) and 1.6) with an additional parameter $q$, i.e.,

$$
p_{1} \int_{a}^{b} \frac{F^{p_{1}}(x)}{x^{q}} d x \leq(b-a)^{p_{1}} \int_{a}^{b} \frac{f^{p_{1}}(x)}{x^{q}} d x-\int_{a}^{b} \frac{(x-a)^{p_{1}}}{x^{q}} f^{p_{1}}(x) d x
$$

and

$$
p_{2} \int_{a}^{b} \frac{F^{p_{2}}(x)}{x^{q}} d x \geq \frac{(b-a)^{p_{2}}}{b^{q}} \int_{a}^{b} f^{p_{2}}(x) d x-\frac{1}{b^{q}} \int_{a}^{b}(x-a)^{p_{2}} f^{p_{2}}(x) d x,
$$

where $f>0$ on $[a, b] \subseteq(0, \infty), 0<p_{2}<1 \leq p_{1}, q>0$ and

$$
F(x)=\int_{a}^{x} f(t) d t, \quad x \in[a, b] .
$$

In this paper, by introducing a monotonous function, we establish further generalizations of the above inequalities (1.4), (1.5), (1.6), 1.7) and (1.8).

\section{Lemmas}

The inequality (2.1) below is known in the literature as Hölder integral inequality (see, e.g., [12], [13], [25] and [26]), which will be used as a main tool for establishing required inequalities in the next section.

Lemma 2.1 (Hölder inequality). Let $p>1$ and $\frac{1}{p}+\frac{1}{q}=1$. If $f$ and $g$ are nonnegative integrable functions defined on $[a, b]$, then

$$
\int_{a}^{b} f(x) g(x) d x \leq\left(\int_{a}^{b} f^{p}(x) d x\right)^{\frac{1}{p}}\left(\int_{a}^{b} g^{q}(x) d x\right)^{\frac{1}{q}}
$$

with equality holding if and only if $A f^{p}(x)=B g^{q}(x)$ almost everywhere, where $A$ and $B$ are constants.

A reversed form of inequality (2.1) is stated in the following lemma.

Lemma 2.2 (Reverse Hölder inequality). Let $0<p<1$ and $\frac{1}{p}+\frac{1}{q}=1$. If $f$ and $g$ are positive integrable functions defined on $[a, b]$, then

$$
\int_{a}^{b} f(x) g(x) d x \geq\left(\int_{a}^{b} f^{p}(x) d x\right)^{\frac{1}{p}}\left(\int_{a}^{b} g^{q}(x) d x\right)^{\frac{1}{q}}
$$

with equality holding if and only if $A f^{p}(x)=B g^{q}(x)$ almost everywhere, where $A$ and $B$ are constants. 
Proof. From $0<p<1$ and $\frac{1}{p}+\frac{1}{q}=1$, we conclude that

$$
\frac{-p}{q}+p=1, \quad 0<p<1, \quad 0<\frac{-p}{q}<1,
$$

which, along with the Hölder inequality (2.1), leads us to

$$
\left(\int_{a}^{b} f(x) g(x) d x\right)^{p}\left(\int_{a}^{b} g^{q}(x) d x\right)^{-\frac{p}{q}} \geq \int_{a}^{b} f^{p}(x) d x .
$$

Simplifying the above inequality yields immediately the reverse Hölder inequality 2.2 .

\section{Main Results}

Throughout this section, functions are assumed to be integrable. Firstly, we give a generalization of the Levinson's inequality (1.4), as follows

Theorem 3.1. Let $\eta$ be a nonnegative real number, and let $f \geq 0$ and $g>0$ on $[a, b] \subseteq(0, \infty)$ such that $(x-a+\eta) / g(x)$ is non-increasing. Define

$$
F(x)=\int_{a}^{x} f(t) d t, \quad x \in[a, b] .
$$

Then for $p>1$, we have the inequality

$$
\int_{a}^{b}\left(\frac{F(x)}{g(x)}\right)^{p} d x \leq\left(\frac{p}{p-1}\right)^{p} \int_{a}^{b}\left((x-a+\eta) \frac{f(x)}{g(x)}\right)^{p} d x .
$$

Proof. Using the Hölder inequality (2.1), and then invoking the assumption condition that $(x-a) / g(x)$ is non-increasing on $[a, b]$, it follows that

$$
\begin{aligned}
\int_{a}^{b}\left(\frac{F(x)}{g(x)}\right)^{p} d x & =\int_{a}^{b} g^{-p}(x)\left(\int_{a}^{x} f(t) d t\right)^{p} d x \\
& \leq \int_{a}^{b} g^{-p}(x)\left(\left(\int_{a}^{x}(t-a)^{1-\frac{1}{p}} f^{p}(t) d t\right)^{\frac{1}{p}}\left(\int_{a}^{x}(t-a)^{-\frac{1}{p}} d t\right)^{1-\frac{1}{p}}\right)^{p} d x \\
& =\int_{a}^{b} g^{-p}(x)\left(\int_{a}^{x}(t-a)^{1-\frac{1}{p}} f^{p}(t) d t\right)\left(\int_{a}^{x}(t-a)^{-\frac{1}{p}} d t\right)^{p-1} d x \\
& =\frac{1}{\left(1-\frac{1}{p}\right)^{p-1}} \int_{a}^{b} d x \int_{a}^{x}(x-a)^{\left(1-\frac{1}{p}\right)(p-1)} g^{-p}(x)(t-a)^{1-\frac{1}{p}} f^{p}(t) d t \\
& =\frac{1}{\left(1-\frac{1}{p}\right)^{p-1}} \int_{a}^{b} d t \int_{t}^{b}(x-a)^{\left(1-\frac{1}{p}\right)(p-1)} g^{-p}(x)(t-a)^{1-\frac{1}{p}} f^{p}(t) d x \\
& =\frac{1}{\left(1-\frac{1}{p}\right)^{p-1}} \int_{a}^{b} d t \int_{t}^{b}(x-a)^{\frac{1}{p}-2}\left(\frac{x-a}{g(x)}\right)^{p}(t-a)^{1-\frac{1}{p}} f^{p}(t) d x \\
& \leq \frac{1}{\left(1-\frac{1}{p}\right)^{p-1}} \int_{a}^{b} d t \int_{t}^{b}(x-a)^{\frac{1}{p}-2}\left(\frac{x-a+\eta}{g(x)}\right)^{p}(t-a)^{1-\frac{1}{p}} f^{p}(t) d x \\
& \leq \frac{1}{\left(1-\frac{1}{p}\right)^{p-1}} \int_{a}^{b}\left(\frac{t-a+\eta}{g(t)}\right)^{p}(t-a)^{1-\frac{1}{p}} f^{p}(t) d t \int_{t}^{b}(x-a)^{\frac{1}{p}-2} d x
\end{aligned}
$$




$$
\begin{aligned}
& =\frac{1}{\left(1-\frac{1}{p}\right)^{p-1}} \int_{a}^{b}\left(\frac{t-a+\eta}{g(t)}\right)^{p} \frac{(b-a)^{\frac{1}{p}-1}-(t-a)^{\frac{1}{p}-1}}{\frac{1}{p}-1}(t-a)^{1-\frac{1}{p}} f^{p}(t) d t \\
& =\frac{1}{\left(1-\frac{1}{p}\right)^{p}} \int_{a}^{b}\left(\frac{t-a+\eta}{g(t)}\right)^{p}\left(1-\frac{(t-a)^{1-\frac{1}{p}}}{(b-a)^{1-\frac{1}{p}}}\right) f^{p}(t) d t \\
& \leq\left(\frac{p}{p-1}\right)^{p} \int_{a}^{b}\left((t-a+\eta) \frac{f(t)}{g(t)}\right)^{p} d t,
\end{aligned}
$$

which implies the desired inequality 3.1 asserted by Theorem 3.1

In particular, if we put in Theorem 3.1 with

$$
g(x)=x-a+\eta, \quad 0<\eta<a, \quad x \in[a, b],
$$

we get the following result.

Corollary 3.2. Let $f$ be a nonnegative function on $[a, b] \subseteq(0, \infty)$, and let $0<\eta<a$. Define

$$
F(x)=\int_{a}^{x} f(t) d t, \quad x \in[a, b] .
$$

Then for $p>1$, we have the inequality

$$
\int_{a}^{b}\left(\frac{F(x)}{x-a+\eta}\right)^{p} d x \leq\left(\frac{p}{p-1}\right)^{p} \int_{a}^{b} f^{p}(x) d x
$$

Remark 3.3. It is clear that, for $0<\eta<a$, the following inequality holds true

$$
\int_{a}^{b}\left(\frac{F(x)}{x}\right)^{p} d x \leq \int_{a}^{b}\left(\frac{F(x)}{x-a+\eta}\right)^{p} d x \leq\left(\frac{p}{p-1}\right)^{p} \int_{a}^{b} f^{p}(x) d x .
$$

Accordingly, the inequality $(3.2)$ in Corollary 3.2 implies the Levinson's inequality (1.4) which we have mentioned in Section 1 .

In addition, choosing $\eta=0$ in Theorem 3.1, we obtain,

Corollary 3.4. Let $f \geq 0$ and $g>0$ on $[a, b] \subseteq(0, \infty)$ such that $(x-a) / g(x)$ is non-increasing. Define

$$
F(x)=\int_{a}^{x} f(t) d t, \quad x \in[a, b] .
$$

Then for $p>1$, we have the inequality

$$
\int_{a}^{b}\left(\frac{F(x)}{g(x)}\right)^{p} d x \leq\left(\frac{p}{p-1}\right)^{p} \int_{a}^{b}\left((x-a) \frac{f(x)}{g(x)}\right)^{p} d x .
$$

Next, we establish the generalized versions of the Sulaiman's inequalities (1.5), 1.6) and Sroysang's inequalities (1.7), (1.8).

Theorem 3.5. Let $q>0$, and let $f, g>0$ on $[a, b] \subseteq(0, \infty)$ such that $g$ is non-decreasing. Define 


$$
F(x)=\int_{a}^{x} f(t) d t, \quad x \in[a, b]
$$

(i) If $p \geq 1$, then

$$
p \int_{a}^{b} \frac{F^{p}(x)}{g^{q}(x)} d x \leq(b-a)^{p} \int_{a}^{b} \frac{f^{p}(x)}{g^{q}(x)} d x-\int_{a}^{b}(x-a)^{p} \frac{f^{p}(x)}{g^{q}(x)} d x .
$$

(ii) If $0<p<1$, then

$$
p \int_{a}^{b} \frac{F^{p}(x)}{g^{q}(x)} d x \geq(b-a)^{p} \int_{a}^{b} \frac{f^{p}(x)}{g^{q}(b)} d x-\int_{a}^{b}(x-a)^{p} \frac{f^{p}(x)}{g^{q}(b)} d x .
$$

Proof. Case (i) $p \geq 1$.

By using the Hölder's inequality (2.1) together with the assumption that $g$ is non-decreasing, we deduce that

$$
\begin{aligned}
\int_{a}^{b} \frac{F^{p}(x)}{g^{q}(x)} d x & =\int_{a}^{b} g^{-q}(x)\left(\int_{a}^{x} f(t) d t\right)^{p} d x \\
& \leq \int_{a}^{b} g^{-q}(x)\left(\left(\int_{a}^{x} f^{p}(t) d t\right)^{\frac{1}{p}}\left(\int_{a}^{x} d t\right)^{\frac{p-1}{p}}\right)^{p} d x \\
& =\int_{a}^{b} g^{-q}(x)\left(\left(\int_{a}^{x} f^{p}(t) d t\right)^{\frac{1}{p}}(x-a)^{\frac{p-1}{p}}\right)^{p} d x \\
& =\int_{a}^{b} g^{-q}(x)\left(\int_{a}^{x} f^{p}(t) d t\right)(x-a)^{p-1} d x \\
& =\int_{a}^{b} d x \int_{a}^{x} g^{-q}(x)(x-a)^{p-1} f^{p}(t) d t \\
& =\int_{a}^{b} d t \int_{t}^{b} g^{-q}(x)(x-a)^{p-1} f^{p}(t) d x \\
& \leq \int_{a}^{b} d t \int_{t}^{b} g^{-q}(t)(x-a)^{p-1} f^{p}(t) d x \\
& =\int_{a}^{b} g^{-q}(t) f^{p}(t)\left(\int_{t}^{b}(x-a)^{p-1} d x\right) d t \\
& =\int_{a}^{b} g^{-q}(t) f^{p}(t)\left(\frac{(b-a)^{p}-(t-a)^{p}}{p}\right) d t \\
& =\frac{1}{p}\left((b-a)^{p} \int_{a}^{b} \frac{f^{p}(t)}{g^{q}(t)} d t-\int_{a}^{b}(t-a)^{p} \frac{f^{p}(t)}{g^{q}(t)} d t\right) .
\end{aligned}
$$

Hence, the desired inequality $(3.5)$ is proved.

Case (ii) $0<p<1$.

By utilizing the reverse Hölder's inequality 2.2 and by the assumption that $g$ is non-decreasing, we obtain that 


$$
\begin{aligned}
\int_{a}^{b} \frac{F^{p}(x)}{g^{q}(x)} d x & =\int_{a}^{b} g^{-q}(x)\left(\int_{a}^{x} f(t) d t\right)^{p} d x \\
& \geq \int_{a}^{b} g^{-q}(x)\left(\left(\int_{a}^{x} f^{p}(t) d t\right)^{\frac{1}{p}}\left(\int_{a}^{x} d t\right)^{\frac{p-1}{p}}\right)^{p} d x \\
& =\int_{a}^{b} g^{-q}(x)\left(\left(\int_{a}^{x} f^{p}(t) d t\right)^{\frac{1}{p}}(x-a)^{\frac{p-1}{p}}\right)^{p} d x \\
& =\int_{a}^{b} g^{-q}(x)\left(\int_{a}^{x} f^{p}(t) d t\right)(x-a)^{p-1} d x \\
& =\int_{a}^{b} d x \int_{a}^{x} g^{-q}(x)(x-a)^{p-1} f^{p}(t) d t \\
& =\int_{a}^{b} d t \int_{t}^{b} g^{-q}(x)(x-a)^{p-1} f^{p}(t) d x \\
& \geq \int_{a}^{b} d t \int_{t}^{b} g^{-q}(b)(x-a)^{p-1} f^{p}(t) d x \\
& =g^{-q}(b) \int_{a}^{b} f^{p}(t)\left(\int_{t}^{b}(x-a)^{p-1} d x\right) d t \\
& =g^{-q}(b) \int_{a}^{b} f^{p}(t)\left(\frac{(b-a)^{p}-(t-a)^{p}}{p} d t\right. \\
& =\frac{g^{-q}(b)}{p}\left((b-a)^{p} \int_{a}^{b} f^{p}(t) d t-\int_{a}^{b}(t-a)^{p} f^{p}(t) d t\right) \\
& =\frac{1}{p}\left((b-a)^{p} \int_{a}^{b} \frac{f^{p}(t)}{g^{q}(b)} d t-\int_{a}^{b}(t-a)^{p} \frac{f^{p}(t)}{g^{q}(b)} d t\right) .
\end{aligned}
$$

This proves the required inequality (3.6). The proof of Theorem 3.5 is completed.

Finally, we show some consequences of the Theorem 3.5 .

Putting $g(x)=x$ in Theorem 3.5, we get the Sroysang's inequalities (1.7) and (1.8) stated in Section 1. Also, we can deduce the Sulaiman's inequalities (1.5) and (1.6) from the Theorem 3.5 as a special case of $g(x)=x$ and $q=p$.

Moreover, if we take $q=p$ in Theorem 3.5, we obtain following corollary.

Corollary 3.6. Let $f, g>0$ on $[a, b] \subseteq(0, \infty)$ such that $g$ is non-decreasing. Define

$$
F(x)=\int_{a}^{x} f(t) d t, \quad x \in[a, b] .
$$

(i) If $p \geq 1$, then

$$
p \int_{a}^{b}\left(\frac{F(x)}{g(x)}\right)^{p} d x \leq(b-a)^{p} \int_{a}^{b}\left(\frac{f(x)}{g(x)}\right)^{p} d x-\int_{a}^{b}(x-a)^{p}\left(\frac{f(x)}{g(x)}\right)^{p} d x .
$$

(ii) If $0<p<1$, then

$$
p \int_{a}^{b}\left(\frac{F(x)}{g(x)}\right)^{p} d x \geq(b-a)^{p} \int_{a}^{b}\left(\frac{f(x)}{g(b)}\right)^{p} d x-\int_{a}^{b}(x-a)^{p}\left(\frac{f(x)}{g(b)}\right)^{p} d x .
$$

Choosing $g(x)=x-a+\eta(\eta>0)$ in Theorem 3.5, we get following corollary. 
Corollary 3.7. Let $f$ be a positive valued function on $[a, b] \subseteq(0, \infty)$, and let $q>0, \eta>0$. Define

$$
F(x)=\int_{a}^{x} f(t) d t, \quad x \in[a, b] .
$$

(i) If $p \geq 1$, then

$$
p \int_{a}^{b} \frac{F^{p}(x)}{(x-a+\eta)^{q}} d x \leq(b-a)^{p} \int_{a}^{b} \frac{f^{p}(x)}{(x-a+\eta)^{q}} d x-\int_{a}^{b} \frac{(x-a)^{p}}{(x-a+\eta)^{q}} f^{p}(x) d x .
$$

(ii) If $0<p<1$, then

$$
p \int_{a}^{b} \frac{F^{p}(x)}{(x-a+\eta)^{q}} d x \geq(b-a)^{p} \int_{a}^{b} \frac{f^{p}(x)}{(b-a+\eta)^{q}} d x-\int_{a}^{b} \frac{(x-a)^{p}}{(b-a+\eta)^{q}} f^{p}(x) d x .
$$

Further, setting $q=p$ in Corollary 3.7 yields the following inequalities.

Corollary 3.8. Let $f$ be a positive valued function on $[a, b] \subseteq(0, \infty)$, and let $\eta>0$. Define

$$
F(x)=\int_{a}^{x} f(t) d t, \quad x \in[a, b] .
$$

(i) If $p \geq 1$, then

$$
p \int_{a}^{b}\left(\frac{F(x)}{x-a+\eta}\right)^{p} d x \leq(b-a)^{p} \int_{a}^{b}\left(\frac{f(x)}{x-a+\eta}\right)^{p} d x-\int_{a}^{b}\left(\frac{x-a}{x-a+\eta}\right)^{p} f^{p}(x) d x .
$$

(ii) If $0<p<1$, then

$$
p \int_{a}^{b}\left(\frac{F(x)}{x-a+\eta}\right)^{p} d x \geq(b-a)^{p} \int_{a}^{b}\left(\frac{f(x)}{b-a+\eta}\right)^{p} d x-\int_{a}^{b}\left(\frac{x-a}{b-a+\eta}\right)^{p} f^{p}(x) d x .
$$

Remark 3.9. Consider the function

$$
g(x)=\eta+\int_{a}^{x} f(t) d t, \quad x \in[a, b]
$$

where $\eta>0$ and $f>0$ for $x \in[a, b]$.

It is easy to observe that

$$
g^{\prime}(x)=f(x)>0
$$

for all $x \in[a, b]$. This implies that $g$ is increasing on $[a, b]$.

Thus, one can apply the function

$$
g(x)=\eta+\int_{a}^{x} f(t) d t, \quad x \in[a, b]
$$

to the Corollary 3.6 and obtain the following results

Corollary 3.10. Let $f$ be a positive integrable function on $[a, b] \subseteq(0, \infty)$.

(i) If $p \geq 1$, then

$$
\int_{a}^{b}\left(\int_{a}^{x} f(t) d t\right)^{p}\left(\eta+\int_{a}^{x} f(t) d t\right)^{-p} d x \leq \int_{a}^{b} \frac{(b-a)^{p}-(x-a)^{p}}{p}\left(\frac{\eta}{f(x)}+\int_{a}^{x} \frac{f(t)}{f(x)} d t\right)^{-p} d x
$$

(ii) If $0<p<1$, then

$$
\int_{a}^{b}\left(\int_{a}^{x} f(t) d t\right)^{p}\left(\eta+\int_{a}^{x} f(t) d t\right)^{-p} d x \geq \int_{a}^{b} \frac{(b-a)^{p}-(x-a)^{p}}{p}\left(\frac{\eta}{f(x)}+\int_{a}^{b} \frac{f(t)}{f(x)} d t\right)^{-p} d x
$$


In particular, if we take the limit as $\eta \rightarrow 0$ in the inequality (3.14), we get the following inequality

$$
\int_{a}^{b} \frac{(b-a)^{p}-(x-a)^{p}}{p}\left(\int_{a}^{b} \frac{f(t)}{f(x)} d t\right)^{-p} d x \leq b-a,
$$

where $0<p<1$ and $f$ is a positive integrable function on $[a, b] \subseteq(0, \infty)$.

\section{Conflict of interests}

The authors declare that there is no conflict of interests regarding the publication of this article.

\section{Authors' contributions}

All authors contributed equally and significantly in this paper. All authors read and approved the final manuscript.

\section{Acknowledgements}

The present investigation was supported by the National Training Programs of China for Undergraduate Innovation and Entrepreneurship (No.201411312002).

\section{References}

[1] Y. Deng, S. Wu, D. He, A sharpened version of Hardy's inequality for parameter $p=5 / 4$, J. Inequal. Appl., 2013 (2013), 13 pages. 1

[2] T. Ekholm, H. Kovarik, A. Laptev, Hardy inequalities for $p$-Laplacians with Robin boundary conditions, Nonlinear Anal., 128 (2015), 365-379. 1

[3] G. H. Hardy, Note on a theorem of Hilbert, Math. Z., 6 (1920), 314-317. 1

[4] G. H. Hardy, Note on some points in the integral calculus, Messenger Math., 57 (1928), 12-16. 1

[5] G. H. Hardy, J. E. Littlewood, G. Pólya, Inequalities, Cambridge University Press, Cambridge, (1952). 1

[6] D. H. Hong, A sharp Hardy-type inequality of Sugeno integrals, Appl. Math. Comput., 217 (2010), 437-440. 1

[7] M. M. Iddrisu, A. C. Okpoti, A. K. Gbolagade, Some proofs of the classical integral Hardy inequality, Korean J. Math., 22 (2014), 407-417.1

[8] A. Kufner, L. Maligranda, L. E. Persson, The prehistory of the Hardy inequality, Amer. Math. Monthly, 113 (2006), 715-732. 1

[9] N. Levinson, Generalizations of an inequality of Hardy, Duke Math. J., 31 (1964), 389-394. 1

[10] T. Leng, Y. Feng, On Hardy-type integral inequalities, Appl. Math. Mech., 34 (2014), 1297-1304. 1

[11] D. Lukkassen, L. E. Persson, S. Samko, P. Wall, Weighted Hardy-type inequalities in variable exponent Morrey-type spaces, J. Funct. Spaces Appl., 2013 (2013), 11 pages. 1

[12] D. S. Mitrinović, J. E. Pečarić, A. M. Fink, Inequalities Involving Functions and Their Integrals and Derivatives, Kluwer Academic Publishers Group, Dordrecht, (1991). 1, 2

[13] D. S. Mitrinović, J. E. Pečarić, A. M. Fink, Classical and New Inequalities in Analysis, Kluwer Academic Publishers Group, Dordrecht, (1993). 1,2

[14] J. A. Oguntuase, L. E. Persson, Hardy-type inequalities via convexity-the journey so far, Aust. J. Math. Anal. Appl., 7 (2010), 19 pages. 1

[15] J. A. Oguntuase, L. E. Persson, N. Samko, Some Hardy-type inequalities with broken exponent, J. Math. Inequal., 8 (2014), 405-416. 1

[16] J. E. Pečarić, F. Proschan, Y. L. Tong, Convex functions, partial orderings, and statistical applications, Academic Press, Boston, (1992). 1

[17] J. M. Rakotoson, New Hardy inequalities and behaviour of linear elliptic equations, J. Funct. Anal., 263 (2012), 2893-2920. 1

[18] I. Skrzypczak, Hardy-type inequalities derived from p-harmonic problems, Nonlinear Anal., 93 (2013), 30-50. 1

[19] M. Z. Sarikaya, H. Yildirim, Some Hardy type integral inequalities, JIPAM. J. Inequal. Pure Appl. Math., 7 (2006), 5 pages. 1

[20] W. T. Sulaiman, Some Hardy type integral inequalities, Appl. Math. Lett., 25 (2012), 520-525. 1

[21] W. T. Sulaiman, Reverses of Minkowski's, Hölder's, and Hardy's integral inequalities, Int. J. Mod. Math. Sci., 1 (2012), 14-24. 1 
[22] B. Sroysang, A generalization of some integral inequalities similar to Hardy's inequality, Math. Aeterna, 3 (2013), 593-596. 1

[23] B. Sroysang, More on some Hardy type integral inequalities, J. Math. Inequal., 8 (2014), 497-501. 1

[24] J. Tian, Y. X. Zhou, Refinements of Hardy-Type Inequalities, Abstr. Appl. Anal., 2013 (2013), 8 pages. 1

[25] S. Wu, Generalization of a sharp Hölder's inequality and its application, J. Math. Anal. Appl., 332 (2007), 741-750. 2

[26] S. Wu, A new sharpened and generalized version of Hölder's inequality and its applications, Appl. Math. Comput., 197 (2008), 708-714. 2

[27] Q. Yang, D. Su, Y. Kong, Improved Hardy inequalities for Grushin operators, J. Math. Anal. Appl., 424 (2015), 321-343. 1

[28] L. Zhongxue, G. Youcai, W. Yuxiang, Note on the Carleman's inequality and Hardy's inequality, Comput. Math. Appl., 59 (2010), 94-97.1. 